\title{
Global Dynamics of a New Huanglongbing Transmission Model with Quarantine Measures
}

\author{
Chunmei Zeng1, Yujiang Liu', Shuixian Yan², Wenyan Fu² \\ ${ }^{1}$ Key Laboratory of Jiangxi Province for Numerical Simulation and Emulation Techniques, Ganzhou, China \\ ${ }^{2}$ Gannan Normal University, Ganzhou, China \\ Email: gnsylyj@126.com
}

How to cite this paper: Zeng, C.M., Liu, Y.J., Yan, S.X. and Fu, W.Y. (2022) Global Dynamics of a New Huanglongbing Transmission Model with Quarantine Measures. Journal of Applied Mathematics and Physics, $10,360-371$.

https://doi.org/10.4236/jamp.2022.102028

Received: January 8, 2022

Accepted: February 18, 2022

Published: February 21, 2022

Copyright $\odot 2022$ by author(s) and Scientific Research Publishing Inc. This work is licensed under the Creative Commons Attribution International License (CC BY 4.0).

http://creativecommons.org/licenses/by/4.0/

\begin{abstract}
Incorporating plant-dependent saturated reproduction function, chemical insecticide, and its resistance, a plant-vector-virus model which describes the spread of infection throughout the plant by the insect is investigated in this paper. First of all, the basic reproduction number $\mathcal{R}_{0}$ is obtained by using the method of the next generation matrix, and the existence of disease-free equilibrium and endemic equilibrium is examined. Then we show that the disease-free steady state is globally asymptotically stable if $\mathcal{R}_{0}<1$, whereas if $\mathcal{R}_{0}>1$, the system is uniformly persistent and the endemic equilibrium is local stability. Finally, numerical simulation is carried out to illustrate our theoretical results. Our result implies that insecticide resistance has a vital impact on the control of plant diseases.
\end{abstract}

\section{Keywords}

Saturated Reproduction Rate, Vector-Borne Disease Model, Basic Reproduction Number, Sensitivity Analysis

\section{Introduction}

In many plants, viruses are not transmitted only through seeds, but more through insects, such as helminth, leaf butterfly, flying wind, powdery wind, etc. It leads to a series of morphological, physiological and biochemical pathological changes in plants, hinders the process of normal growth and development, and affects human economic benefits, resulting in drastic reductions in yield for crops [1] [2]. It was reported from Food and Agriculture Organization of the United Nations that annually up to 40 percent of global crop production is lost from pests, economic loss is over $\$ 220$ billion [3]. 
Mathematical models of plant vector-borne diseases are usually used to describe, analyze, predict and control the prevalence of plant diseases in details [4] [5] [6] [7] [8]. Holt et al. [4] developed epidemiological model to depict the dynamics of African cassava mosaic virus disease. Theoretical results illustrate that a high virus transmission rate or a large population of vectors could bring on persistent mode. Jeger et al. [8] explored the effect of immigration of vectors and found that it comes into being a negative impact on the efficacy of insecticides for the control of the propagation of the virus.

Recently, many researcher have payed attention to control issues of plant diseases [9] [10] [11] [12] [13]. Venturino et al. [11] formulated a mosaic virus disease model in Jatropha curcas plantations. One interesting result of the model analysis showed that the control policy with minimum use of insecticides can concentrate the oscillatory nature of the vector population and eventually eradicate the disease. Al Basir and Roy [12] formulated a nonlinear mathematical model with roguing and delay. They showed that endemic equilibrium may be occurring bifurcation with periodic solution. Based on the modelling idea of [4] [8], Bokil et al. [13] extended two vectored plant disease models with frequency replanting and abundance replanting. The effects of roguing and insecticide use with a goal of maximizing the healthy plants that are harvested were investigated by using optimal control theory. As we know, constant reproduction rate has been assumed in many models, however, saturated reproduction rate has seldom been considered. Therefore, the main objective of this work is to establish a vectored plant disease model with saturated reproduction function, investigate the dynamics of the model, and evaluate the control strategy.

The paper is organised as follows. In Section 2, mathematical model is presented and the basic reproduction number is formulated. In Section 3, the local and global stability of disease-free equilibrium is studied. In Section 4, the uniformly persistent and the local stability of the endemic equilibrium are analysed. Numerical simulations are carried out to illustrate the analytical results and the effects of control strategy and insecticide resistance are evaluated in Section 5. Finally, a brief discussion concludes the paper.

\section{Model and Preliminaries}

Plant and vector populations are considered in our model. $S(t), E(t)$ and $I(t)$ denote the healthy, the exposed and the infected plant (host) population at time $t$, respectively. Let $N(t)=S(t)+E(t)+I(t)$ be the total population size of host at time $t$. $X(t)$ denotes the vector population. We assume that the growth rate of vector population satisfies plant-dependant saturated reproduction function $\frac{a_{0} N}{k+N}$, where $a_{0}$ is the maximum reproduction rate of vectors, and $\frac{1}{k+N}$ measures the inhibition from the crowding effect of the hosts. Considering saturated reproduction rate, insecticide spraying, and its resistance, we have the following mathematical model: 


$$
\left\{\begin{array}{l}
\frac{\mathrm{d} X(t)}{\mathrm{d} t}=\frac{a_{0} N}{k+N} X-d X-\delta(1-\eta) X-m X^{2}, \\
\frac{\mathrm{d} S(t)}{\mathrm{d} t}=\mu K-\beta S X-\mu S, \\
\frac{\mathrm{d} E(t)}{\mathrm{d} t}=\beta S X-(\mu+\gamma) E, \\
\frac{\mathrm{d} I(t)}{\mathrm{d} t}=\gamma E-(\mu+b) I .
\end{array}\right.
$$

where $\mu$ is the natural death rate of plant population, $\gamma$ is the conversion rate from the exposed to infectious plant, $\delta$ is the killing rate of insecticide, $m$ is the Density-dependent coefficient of vector population, $\eta$ is the resistance effect of the vector to insecticides, and $b$ is the disease-induced death rate of plant. The inoculation rate of healthy plants by vectors is $\beta S X$.

In the following, we mainly discuss the dynamic behavior of model (2.1).

\subsection{Basic Reproduction Number}

For convenience, we denote $a=a_{0} / k, w=1 / k, \mu_{1}=q_{1}+\sigma+\gamma_{1}+\mu_{h}$, $\mu_{2}=q_{2}+\gamma_{2}+\mu_{h}+d$. System (2.1) becomes:

$$
\left\{\begin{array}{l}
\frac{\mathrm{d} X(t)}{\mathrm{d} t}=\frac{a N}{1+w N} X-d_{1} X-m X^{2}, \\
\frac{\mathrm{d} S(t)}{\mathrm{d} t}=\mu K-\beta S X-\mu S, \\
\frac{\mathrm{d} E(t)}{\mathrm{d} t}=\beta S X-\mu_{1} E \\
\frac{\mathrm{d} I(t)}{\mathrm{d} t}=\gamma E-\mu_{2} I .
\end{array}\right.
$$

Define

$$
\Omega=\left\{(X, S, E, I) \in \mathbb{R}_{+}^{4} \mid S+E+I \leq K, X \leq \frac{a K}{m(1+w K)}\right\} .
$$

It can be shown that the set $\Omega$ is invariant.

Lemma 1 . The set $\Omega$ is the positively invariant of system (2.2).

Proof. First, it is easy to obtain the positivity of the solutions of system (2.2). Then we will discuss the boundedness of the solutions of system (2.2).

Adding the last three equations of system (2.2), we have

$$
\frac{\mathrm{d} N(t)}{\mathrm{d} t}=\mu K-\mu N-b I .
$$

Thus, $\limsup _{t \rightarrow \infty} N(t) \leq K$. It follows from the first equations of system (2.2), we have that there exists $T_{1}>0$ such that

$$
\frac{\mathrm{d} X(t)}{\mathrm{d} t} \leq \frac{a K}{1+w K} X-m X^{2}, \text { for } t>T_{1} .
$$

Solving the differential inequality (2.5), we have 


$$
X(t) \leq \frac{\left(\frac{a K}{1+w K}\right) X(0)}{m X(0)+\left(\frac{a K}{1+w K}-m X(0)\right) \mathrm{e}^{-\left(\frac{a K}{1+w K}\right) t}}
$$

Therefore, we have $\limsup _{t \rightarrow \infty} X(t)=\frac{a K}{m(1+w K)}$.

Since $\Omega$ is a positively invariant and globally attractive set for system (2.2). From now on, we restrict the analysis of system (2.2) to the positive invariant set $\Omega$.

Obviously, the disease free equilibrium point (DFE) exists and is given by $P_{0}=(0, K, 0,0)$.

The basic reproductive number denoted by $\mathcal{R}_{0}$, is defined as the average number of secondary infections caused by an infectious individual in a fully susceptible population during its infection period. We shall compute $\mathcal{R}_{0}$ using the next generation operator approach as described by Van den Driessche and Watmough [14] as follows.

Denote the infectious compartments as $x=(X, E, I)^{\mathrm{T}}$. Let

$$
F(x)=\left(F_{1}(x), F_{2}(x), F_{3}(x)\right)^{\mathrm{T}}=\left(\begin{array}{c}
\frac{a N}{1+w N} X \\
\beta S X \\
0
\end{array}\right)
$$

and

$$
V(x)=\left(V_{1}(x), V_{2}(x), V_{3}(x)\right)^{\mathrm{T}}=\left(\begin{array}{c}
d_{1} X+m X^{2} \\
\mu_{1} E \\
\mu_{2} I-\gamma E
\end{array}\right)
$$

represent the rate of appearance of new infection, the transfer rate between compartments, respectively. The Jacobian matrix of $F$ and $V$ with respect to $x$ at $P_{0}$ are given below

$$
\mathcal{F}(x)=\left(\begin{array}{ccc}
\frac{a K}{1+w K} & 0 & 0 \\
\beta K & 0 & 0 \\
0 & 0 & 0
\end{array}\right), \quad \mathcal{V}(x)=\left(\begin{array}{ccc}
d_{1} & 0 & 0 \\
0 & \mu_{1} & 0 \\
0 & -\gamma & \mu_{2}
\end{array}\right) .
$$

Using the next generation matrix approach, the basic reproduction number $\mathcal{R}_{0}$ is the spectral radius of $\mathcal{F} \mathcal{V}^{-1}$, i.e.,

$$
\mathcal{R}_{0}=\frac{a K}{d_{1}(1+w K)}
$$

According to [14], we have following result.

Theorem 2. If $\mathcal{R}_{0}<1$, the disease-free equilibrium $P_{0}$ local asymptotically stable, and if $\mathcal{R}_{0}>1$, the disease-free equilibrium $P_{0}$ is unstable.

\subsection{The Existence of Endemic Equilibrium}

We obtain the endemic equilibrium $P^{*}\left(X^{*}, S^{*}, E^{*}, I^{*}\right)$ by setting the right 
hand side of system (2.2) to zero as following:

$$
\begin{aligned}
& \frac{a N}{1+w N} X-d_{1} X-m X^{2}=0, \\
& \mu K-\beta S X-\mu S=0, \\
& \beta S X-\mu_{1} E=0, \\
& \gamma E-\mu_{2} I=0 .
\end{aligned}
$$

By simple calculation, it follows from the equations of (2.8), we get

$$
\begin{aligned}
& X^{*}=\frac{a N^{*}}{m\left(1+w N^{*}\right)}-d_{1}, \quad I^{*}=\frac{\mu\left(K-N^{*}\right)}{b}, \\
& E^{*}=\frac{\mu \mu_{2}\left(K-N^{*}\right)}{b \gamma}, \quad S^{*}=\frac{m \mu \mu_{1} \mu_{2}\left(1+w N^{*}\right)\left(K-N^{*}\right)}{b \beta \gamma\left(a N^{*}-d_{1} m\left(1+w N^{*}\right)\right)},
\end{aligned}
$$

where $N^{*}=S^{*}+E^{*}+I^{*}$. Substituting $S^{*}, E^{*}, I^{*}$ in (2.9) into $N^{*}=S^{*}+E^{*}+I^{*}$, we have

$$
f\left(N^{*}\right)=a_{0}\left(N^{*}\right)^{2}+a_{1} N^{*}+a_{2}=0
$$

where

$$
\begin{aligned}
& a_{0}=\beta\left(a-w d_{1}\right)\left(b \gamma+\mu\left(\mu_{2}+\gamma\right)\right)+m w \mu \mu_{1} \mu_{2}, \\
& a_{1}=m \mu \mu_{1} \mu_{2}(1-w K)-\mu K \beta\left(\mu_{2}+\gamma\right)\left(a-w d_{1}\right)-d_{1} \beta\left(\gamma(b+\mu)+\mu \mu_{2}\right), \\
& a_{2}=\mu K\left(\beta d_{1}\left(\mu_{2}+\gamma\right)-m \mu_{1} \mu_{2}\right) .
\end{aligned}
$$

It is easy to obtain that $a_{0}>0$ and $\frac{d_{1}}{a-d_{1} w}>0$ if $\mathcal{R}_{0}>1$. In order to ensure the positivity of $X^{*}$, it follows from (2.9), we have

$$
K \geq N \geq \frac{d_{1}}{a-d_{1} w}>0 .
$$

By calculating, we get

$$
\begin{aligned}
& f\left(\frac{d_{1}}{a-d_{1} w}\right)=-\frac{a m \mu \mu_{1} \mu_{2} d_{1}(1+w K)\left(\mathcal{R}_{0}-1\right)}{\left(a-w d_{1}\right)^{2}}<0 \\
& f(K)=r b K \beta d_{1}(1+w K)\left(\mathcal{R}_{0}-1\right)>0
\end{aligned}
$$

From (2.12) and (2.13), we have that Equation (2.10) has a unique positive solution $N^{*}=\frac{-b+\sqrt{b^{2}-4 a c}}{2 a}$ in the interval $\left(\frac{d_{1}}{a-d_{1} w}, K\right)$. Consequently, we can get the following theorem.

Theorem 3. System (2.2) always has a disease-free equilibrium $P_{0}(0, K, 0,0)$. In addition, when $\mathcal{R}_{0}>1$, it also has a unique endemic equilibrium $P^{*}\left(X^{*}, S^{*}, E^{*}, I^{*}\right)$.

\section{Stability of Equilibria}

In this section, we will discuss the global stability of the disease-free equilibrium $P_{0}$ and the local stability of the endemic equilibrium $P^{*}$. 
Theorem 4. If $\mathcal{R}_{0}<1$, the disease-free equilibrium $P_{0}$ of system (2.2) is globally asymptotically stable.

Proof. Let $x=S(t) \in \mathbb{R}$ denote the uninfected compartment and $y=(X(t), E(t), I(t)) \in \mathbb{R}^{3}$ denote all infected compartments. System (2.2) can be rewritten as

$$
\left\{\begin{array}{l}
\frac{\mathrm{d} x}{\mathrm{~d} t}=G(x, y), \\
\frac{\mathrm{d} y}{\mathrm{~d} t}=H(x, y),
\end{array}\right.
$$

where $G(x, y)=\mu K-\beta S X-\mu$, and

$$
H(x, y)=\left[\begin{array}{c}
\frac{a N}{1+w N} X-d_{1} X-m X^{2} \\
\beta S X-\mu_{1} E \\
\gamma E-\mu_{2} I
\end{array}\right], H(x, 0)=0 .
$$

The disease-free equilibrium is now denoted by $P_{0}\left(x^{0}, 0\right)$, where $x^{0}=K$. According to [15], we know that the disease-free equilibrium of system (2.2) is globally asymptotically stable provided that the following two conditions hold:

(H1) For $\frac{\mathrm{d} x}{\mathrm{~d} t}=G(x, 0), x^{0}$ is globally asymptotically stable;

(H2) $H(x, y)=A y-\hat{H}(x, y), \hat{H}(x, y) \geq 0$ for $(x, y) \in \Omega$, where $A=D_{y} H\left(x^{0}, 0\right)$ is an M-matrix.

Clearly, $\frac{\mathrm{d} x}{\mathrm{~d} t}=G(x, 0)=\mu K-\mu S$, and $x^{0}=K$ is globally asymptotically stable.

$$
A=D_{y} H\left(x^{0}, 0\right)=\left[\begin{array}{ccc}
\frac{a K}{1+w K}-d_{1} & 0 & 0 \\
\beta K & -\mu_{1} & 0 \\
0 & \gamma & -\mu_{2}
\end{array}\right]
$$

and

$$
\hat{H}=\left[\begin{array}{c}
\widehat{H_{1}} \\
\widehat{H_{2}} \\
\widehat{H_{3}}
\end{array}\right]=\left[\begin{array}{c}
\left(\frac{a K}{1+w K}-\frac{a N}{1+w N}\right) X+m X^{2} \\
\beta(K-S) X \\
0
\end{array}\right]
$$

It is easy to see that (H1) and (H2) are satisfied if $\mathcal{R}_{0}>1$. Thus we can conclude that $P_{0}$ is globally asymptotically stable for $\mathcal{R}_{0}<1$.

Then, we examine the local stability of the endemic equilibrium $P^{*}$. The Jacobian matrix at the endemic equilibrium $P^{*}\left(S^{*}, E^{*}, I^{*}, X^{*}, Y^{*}\right)$ is

$$
\mathcal{J}^{*}=\left(\begin{array}{cccc}
-m X^{*} & \frac{a X^{*}}{\left(1+w N^{*}\right)^{2}} & 0 & 0 \\
0 & -\mu & 0 & -b \\
\frac{\mu_{1} E^{*}}{X^{*}} & \beta X^{*} & -\beta X^{*}-\mu_{1} & -\beta X^{*} \\
0 & 0 & \gamma & -\mu_{2}
\end{array}\right) .
$$


By calculating, the characteristic equation of matrix $\mathcal{J}^{*}$ is

$$
\lambda^{4}+b_{1} \lambda^{3}+b_{2} \lambda^{2}+b_{3} \lambda+b_{4}=0
$$

where

$$
\begin{aligned}
& A=m X^{*}+\mu, \quad B=m \mu X^{*}, \\
& C=\beta X^{*}+\mu+\mu_{2}, \quad D=\mu_{2}\left(\beta X^{*}+\mu\right)+r \beta X^{*}, \\
& b_{1}=A+C>0, \\
& b_{2}=A C+B+D>0, \\
& b_{3}=A D+B C+b r \beta X^{*}>0, \\
& b_{4}=m \mu_{1} \mu_{2}\left(\beta X^{*}+\mu\right) X^{*}+\frac{a b \gamma \mu_{1} E^{*}}{\left(1+w N^{*}\right)^{2}}>0 .
\end{aligned}
$$

It is easy to get $b_{1} b_{2}-b_{3}=(A+C) A C+A B+C D-b r \beta X^{*}>0$. By Routh-Hurwitz criterion, $P^{*}$ is local asymptotically stable if and only if $\left(b_{1} b_{2}-b_{3}\right) b_{3}-b_{1}^{2} b_{4}>0$. Thus, we obtain the following result.

Theorem 5. If $\mathcal{R}_{0}>1$ and $\left(b_{1} b_{2}-b_{3}\right) b_{3}-b_{1}^{2} b_{4}>0$, the endemic equilibrium $P^{*}$ is locally asymptotically stable.

\section{Uniformly Persistence}

The uniformly persistence of system (2.2) is studied in this section.

Theorem 6. System (2.2) is uniformly persistent provided that $\mathcal{R}_{0}>1$, that is, there exists a constant $\eta_{0}>0$, such that

$$
\liminf _{t \rightarrow \infty} X(t) \geq \eta_{0}, \liminf _{t \rightarrow \infty} S(t) \geq \eta_{0}, \liminf _{t \rightarrow \infty} E(t) \geq \eta_{0}, \liminf _{t \rightarrow \infty} I(t) \geq \eta_{0} .
$$

Proof. Denote the following three sets:

$$
\begin{aligned}
& \chi=\left\{(X, S, E, I) \in R^{4}: X \geq 0, S \geq 0, E \geq 0, I \geq 0\right\}, \\
& \chi_{0}=\left\{(X, S, E, I) \in R^{4}: X>0, S \geq 0, E>0, I>0\right\}, \\
& \partial \chi_{0}=\chi \backslash \chi_{0} .
\end{aligned}
$$

In order to illustrate that system (2.2) is uniformly persistent, we need only to show that $\partial \chi_{0}$ repels uniformly the solutions of $\chi_{0}$.

Set

$$
M_{\partial}=\left\{(X(0), S(0), E(0), I(0)) \in \partial \chi_{0}:(X(t), S(t), E(t), I(t)) \in \partial \chi_{0}, \forall t \geq 0\right\} .
$$

We claim that

$$
M_{\partial}=\{(0, S, E, I), S \geq 0, E \geq 0, I \geq 0\} .
$$

Obviously, $\{(0, S, E, I), S \geq 0, E \geq 0, I \geq 0\} \subseteq M_{\partial}$. Next we want to show $M_{\partial} \backslash\{(0, S, E, I), S \geq 0, E \geq 0, I \geq 0\}=\varnothing$. If it does not hold, there exists a point $\left(X^{0}, S^{0}, E^{0}, I^{0}\right) \in M_{\partial} \backslash\{(0, S, E, I), S \geq 0, E \geq 0, I \geq 0\}$. In the following, we divide into three cases to discuss: (i) $X^{0}>0, E^{0}=0, I^{0}=0$, (ii) $X^{0}>0$, $E^{0}>0, I^{0}=0$, and (iii) $X^{0}>0, E^{0}=0, I^{0}>0$.

For case (i), i.e., $X^{0}>0, E^{0}=0, I^{0}=0$. From the first equation of system 
(2.2), we have $\frac{\mathrm{d} X}{\mathrm{~d} t} \geq-d_{1} X-m X^{2}$, which means that $X(t)>0$ for any $t>0$. It follows from the third equation of system (2.2) that $\left.\frac{\mathrm{d} E}{\mathrm{~d} t}\right|_{t=0}=\beta S(0) X(0)>0$. There exists $\tau_{0}>0$, such that $E(t)>0$, for $t \in\left(0, \tau_{0}\right)$. Let $\tau_{1}=\tau_{0} / 2$, we have $X\left(\tau_{1}\right)>0, S\left(\tau_{1}\right)>0, E\left(\tau_{1}\right)>0$. If $I\left(\tau_{1}\right)>0$, then $\left(X^{0}, S^{0}, E^{0}, I^{0}\right) \notin M_{\partial}$. This is a contradiction. If $I\left(\tau_{1}\right)=0$, we have $\left.\frac{\mathrm{d} I}{\mathrm{~d} t}\right|_{t=\tau_{1}}=\gamma E\left(\tau_{1}\right)>0$, then there exists $\tau_{2} \in\left(\tau_{1}, \tau_{0}\right), I(t)>0$ for all $t \in\left(\tau_{1}, \tau_{2}\right)$. This is a contradiction.

Similarly, we can prove the other cases. Based on the above analysis, we have $M_{\partial} \backslash\{(0, S, E, I), S \geq 0, E \geq 0, I \geq 0\}=\varnothing$. This proves (4.3).

Clearly, $P_{0}(0, K, 0,0)$ is the unique equilibrium of system (2.2) in $M_{\partial}$. We shall prove that $P_{0}(0, K, 0,0)$ repels the solutions in which initial values are taken in $\chi_{0}$.

Since $\mathcal{R}_{0}>1$, we can choose a small enough positive constant $\varepsilon$, satisfied

$$
\frac{a(K-\varepsilon)}{1+w(K-\varepsilon)}-d_{1}=\xi_{1}>2 m \varepsilon .
$$

Suppose $(X(t), S(t), E(t), I(t))$ is the solution of system (2.2) with initial value $\left(X^{0}, S^{0}, E^{0}, I^{0}\right)$ in $\chi_{0}$. Let $\xi_{2}=\varepsilon / 2$. We claim that

$$
\limsup _{t \rightarrow \infty} \max \{X(t), E(t), I(t)\}>\xi_{2}
$$

If (4.5) does not hold, then there exists $T_{1}>0$ such that

$$
X(t) \leq \xi_{2}, E(t) \leq \xi_{2}, I(t) \leq \xi_{2} \text {, for } t>T_{1} .
$$

From (2.4) and (4.6), we have $\frac{\mathrm{d} N(t)}{\mathrm{d} t} \geq \mu K-\mu N-\mu \xi_{2}$. Solving the inequality, we have $N(t) \geq K-\xi_{2}+\mathrm{e}^{-\mu t}\left(N_{0}-\left(K-\xi_{2}\right)\right)$. It means that exists a $T_{2}>T_{1}$, for any $t \geq T_{2}, N(t) \geq K-\varepsilon$. From the first equation of system (2.2) and (4.4), we have $\frac{\mathrm{d} X(t)}{\mathrm{d} t} \geq 2 m \varepsilon X(t)-m X(t)^{2}$. Solving the inequality, we have $X(t) \geq \frac{2 m \varepsilon X\left(T_{2}\right)}{\mathrm{e}^{-2 m \varepsilon\left(t-T_{2}\right)}\left(2 m \varepsilon-m X\left(T_{2}\right)\right)+m X\left(T_{2}\right)}$. Thus, there exists a $T_{3}>T_{2}$, for any $t \geq T_{3}, X(t)>\varepsilon=2 \xi_{2}$. This is a contradiction with (4.6), and the claim holds. Thus, $W^{s}\left(P_{0}\right) \cap \chi_{0}=\varnothing$. Every forward orbit of system (2.2) converges to $P_{0}$. By Theorem 4.6 of [16] we can draw a conclusion that the system (2.2) is uniformly persistent with respect to $\left(\chi_{0}, \partial \chi_{0}\right)$. This completes the proof.

\section{Numerical Simulation}

In this section, numerical experiments are given to verify our theoretical results, and the control measures are evaluated.

The parameters in model (2.2) are chosen as $a=3.5, w=0.5, d=5.5$, $\delta=3.2, \quad \eta=0.4, m=0.03, \quad \mu=0.1, K=100, \quad \beta=0.01, \gamma=0.25, \quad b=0.2$, 
and the initial condition is taken as $X(0)=100, S(0)=80, E(0)=50$, $I(0)=50$. By computing, we get $\mathcal{R}_{0}=0.9249<1$. From Figure 1 , we can observe that the disease will die out.

Let $a=5.4, \delta=6.2$, and other parameters are taken as Figure 1. Thus, $\mathcal{R}_{0}=1.1484>1$, the disease will be uniformly persistent (see Figure 2). Figure 3 shows the time series of the infected host with different initial values. By the numerical simulation, we would give a guess that the endemic equilibrium is global stable when $\mathcal{R}_{0}>1$.

Figure 4 shows the comprehensive effects of insecticide resistance $\eta$ and killing rate of insecticide $\delta$ on the basic reproduction number. Numerical result illustrates that increasing the killing rate $\delta$ results in significant reduction

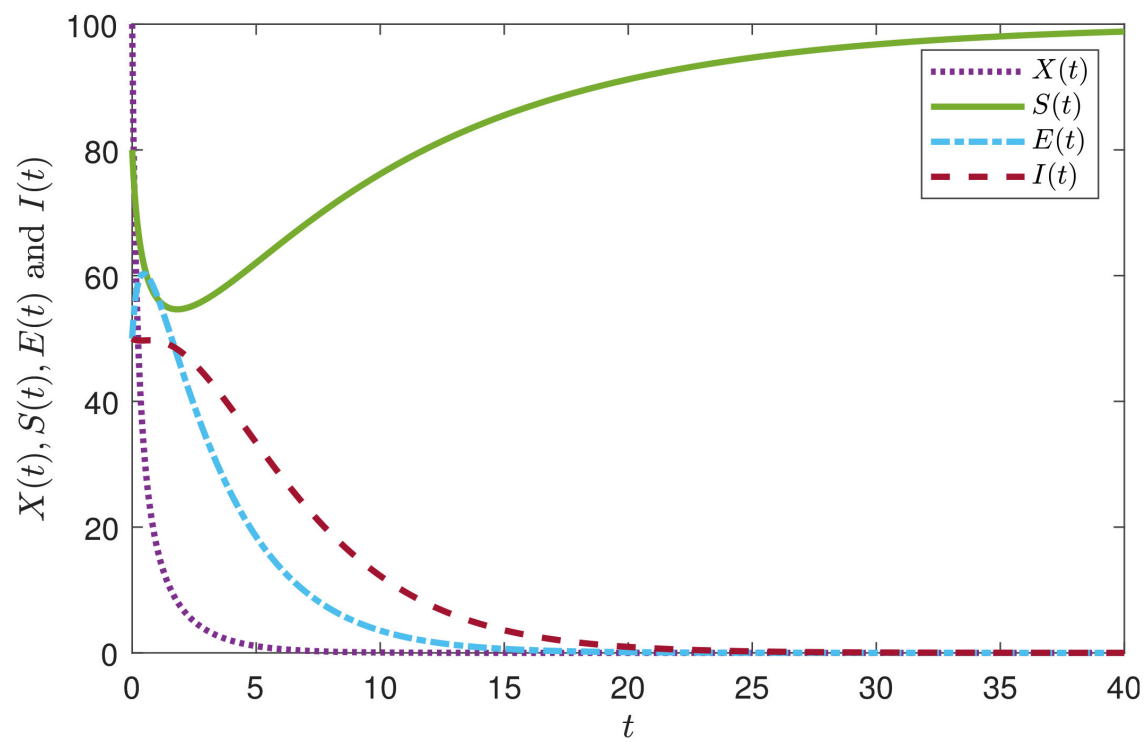

Figure 1. Time series of system (2.2) when $\mathcal{R}_{0}<1$.

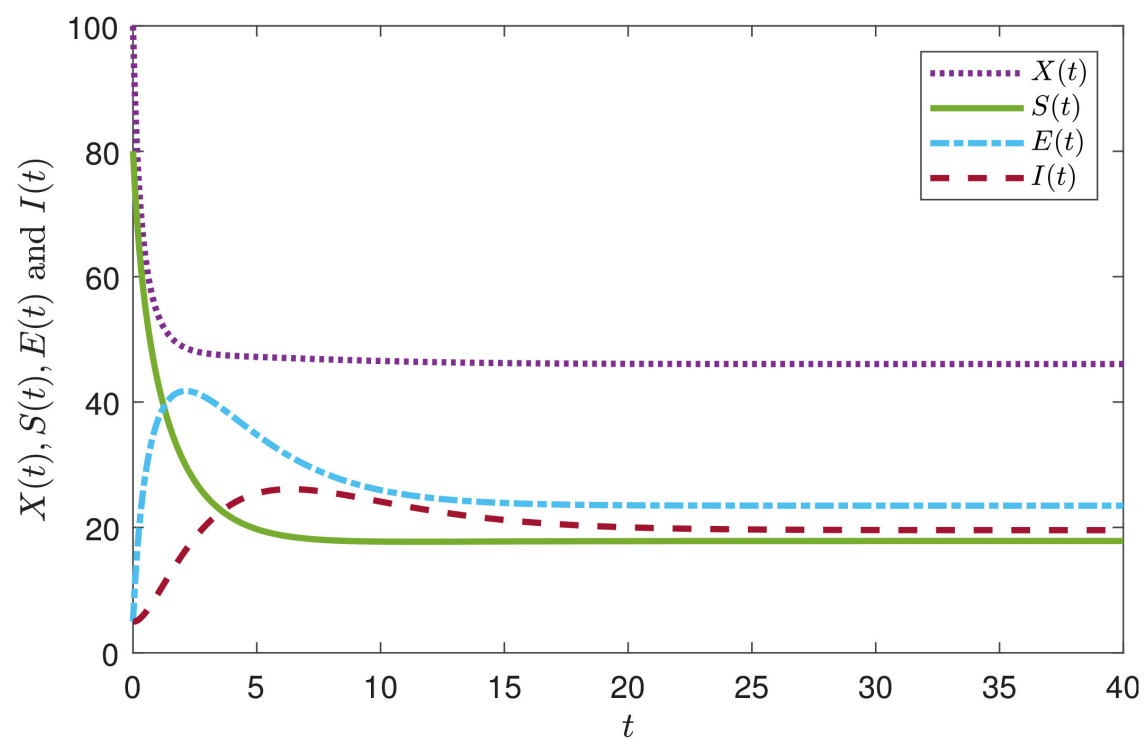

Figure 2. Time series of system (2.2) when $\mathcal{R}_{0}>1$. 


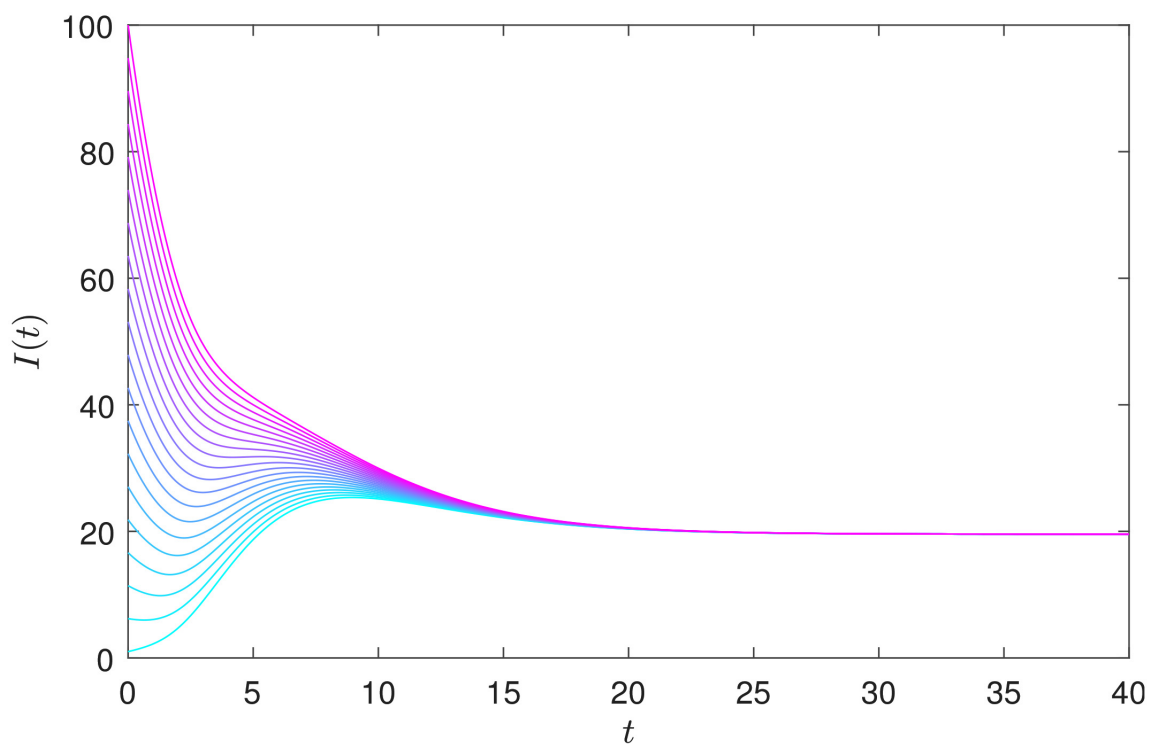

Figure 3. Time series $I(t)$ of system (2.2) with different initial values when $\mathcal{R}_{0}>1$.

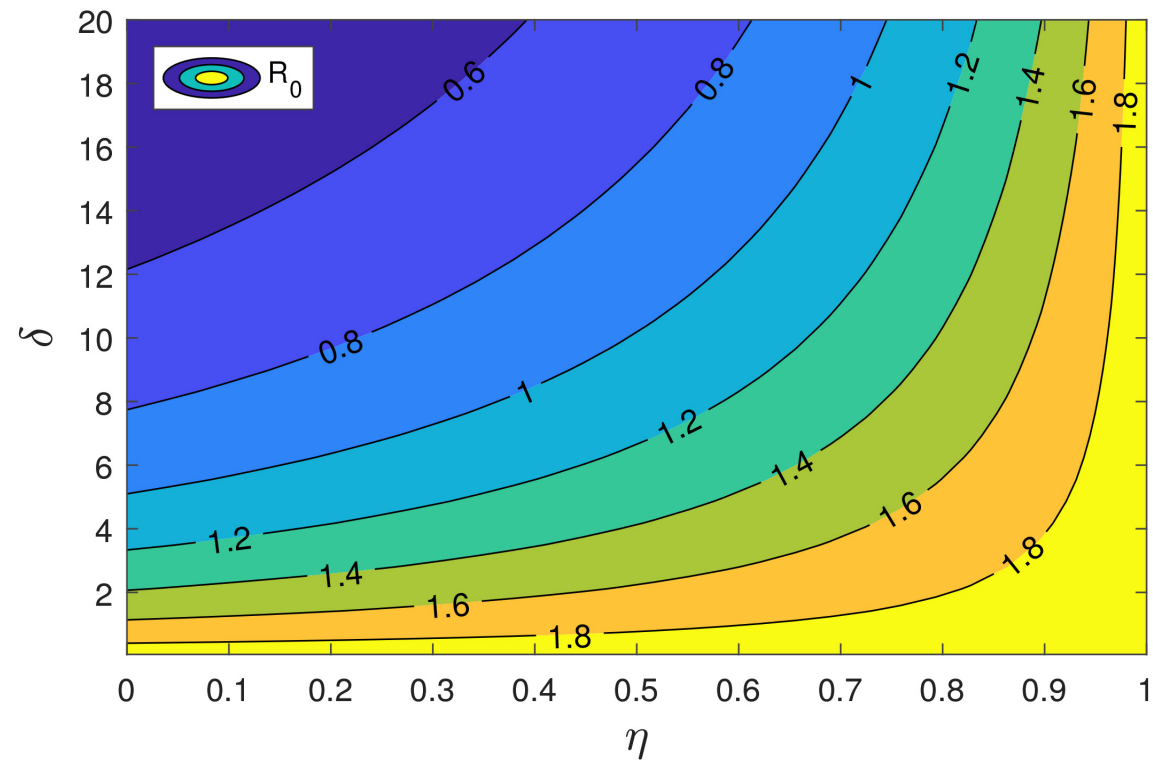

Figure 4. The effects of insecticide resistance $\eta$ and killing rate of pesticides $\delta$ on $\mathcal{R}_{0}$.

in $\mathcal{R}_{0}$ to below unity when the resistance $\eta$ is low. But when resistance $\eta$ is large, increasing the kill rate $\delta$ has no significant effect on $\mathcal{R}_{0}$ increasing the killing rate $\delta$. Therefore, reducing resistance is a valuable method to control disease.

\section{Conclusion}

In this paper, we formulated a plant disease transmission model incorporating plant-dependent saturated reproduction function, insecticides spraying and their resistance. We discussed the stability of equilibria and permanence of the model. We carried out the numerical simulations to illustrate the analytical results and 
evaluate the effect of chemical control and insecticide resistance on the spread of plant disease. Theoretical analysis and numerical simulation results show that the resistance of insecticides has a great impact on the control or elimination of plant diseases. Therefore, timely pesticides switching, biological control, and integrated control are effective measures for prevention and control of plant diseases.

\section{Acknowledgements}

This research was supported by the Natural Science Foundation of China (11961003, 12161005) and the Education Department of Jiangxi Province (GJJ190740, GJJ201406).

\section{Conflicts of Interest}

The authors declare no conflicts of interest regarding the publication of this paper.

\section{References}

[1] Maramorosch, K. (1955) Multiplication of Plant Viruses in Insect Vectors. In: Advances in Virus Research, Volume 3, Elsevier, Amsterdam, 221-249. https://doi.org/10.1016/S0065-3527(08)60637-5

[2] Loebenstein, G. (2008) Plant Virus Diseases: Economic Aspects. In: Mahy, B.W.J. and Van Regenmortel, M.H.V., Eds., Encyclopedia of Virology, Third Edition, Elsevier, Amsterdam, 197-201. https://doi.org/10.1016/B978-012374410-4.00470-2

[3] FAO (2021) Climate Change Fans Spread of Pests and Threatens Plants and Crops. https://www.fao.org/newsroom/detail/Climatechange-fans-spread-of-pests-and-threatens-plants-and-crops-new-FAO-study/en

[4] Holt, J., Jeger, M., Thresh, J. and Otim-Nape, G. (1997) An Epidemiological Model Incorporating Vector Population Dynamics Applied to African Cassava Mosaic Virus Disease. Journal of Applied Ecology, 34, 793-806. https://doi.org/10.2307/2404924

[5] Xia, L., Gao, S., Zou, Q. and Wang, J. (2013) Analysis of a Nonautonomous Plant Disease Model with Latent Period. Applied Mathematics and Computation, 223, 147-159. https://doi.org/10.1016/j.amc.2013.08.011

[6] Buonomo, B. and Cerasuolo, M. (2014) Stability and Bifurcation in Plant-Pathogens Interactions. Applied Mathematics and Computation, 232, 858-871. https://doi.org/10.1016/j.amc.2014.01.127

[7] Zhang, Z.H. and Suo, Y.H. (2014) Stability and Sensitivity Analysis of a Plant Disease Model with Continuous Cultural Control Strategy. Journal of Applied Mathematics, 2014, Article ID: 207959. https://doi.org/10.1155/2014/207959

[8] Jeger, M., Holt, J., Van Den Bosch, F. and Madden, L. (2004) Epidemiology of Insect-Transmitted Plant Viruses: Modelling Disease Dynamics and Control Interventions. Physiological Entomology, 29, 291-304. https://doi.org/10.1111/j.0307-6962.2004.00394.x

[9] Lv, H., Fei, L., Yuan, Z. and Zhang, F. (2018) Global Dynamic Analysis of a Vector-Borne Plant Disease Model with Discontinuous Treatment. Applied Mathematics, 9, 496-511. https://doi.org/10.4236/am.2018.95036 
[10] Wang, J., Feng, F., Guo, Z., Lv, H. and Wang, J. (2019) Threshold Dynamics of a Vector-Borne Epidemic Model for Huanglongbing with Impulsive Control. Applied Mathematics, 10, 196-211. https://doi.org/10.4236/am.2019.104015

[11] Venturino, E., Roy, P.K., Al Basir, F. and Datta, A. (2016) A Model for the Control of the Mosaic Virus Disease in Jatropha curcas Plantations. Energy, Ecology and Environment, 1, 360-369. https://doi.org/10.1007/s40974-016-0033-8

[12] Al Basir, F. and Roy, P.K. (2018) Dynamics of Mosaic Disease with Roguing and Delay in Jatropha curcas Plantations. Journal of Applied Mathematics and Computing, 58, 1-31. https://doi.org/10.1007/s12190-017-1131-2

[13] Bokil, V.A., Allen, L., Jeger, M.J. and Lenhart, S. (2019) Optimal Control of a Vectored Plant Disease Model for a Crop with Continuous Replanting. Journal of Biological Dynamics, 13, 325-353. https://doi.org/10.1080/17513758.2019.1622808

[14] Van den Driessche, P. and Watmough, J. (2002) Reproduction Numbers and Sub-Threshold Endemic Equilibria for Compartmental Models of Disease Transmission. Mathematical Biosciences, 180, 29-48.

https://doi.org/10.1016/S0025-5564(02)00108-6

[15] Castillo-Chavez, C., Feng, Z., Huang, W., et al. (2002) On the Computation of r0 and Its Role in Global Stability. IMA Volumes in Mathematics and Its Applications, 125, 229-250. https://doi.org/10.1007/978-1-4757-3667-0_13

[16] Thieme, H.R. (1993) Persistence under Relaxed Point-Dissipativity with Application to an Endemic Model. SIAM Journal on Mathematical Analysis, 24, 407-435. https://doi.org/10.1137/0524026 